\title{
IMPLICAÇÕES DO SISTEMA DE INFORMAÇÃO DE SAÚDE NA ESTRATÉGIA DE SAÚDE DA FAMÍLIA: UMA REVISÃO INTEGRATIVA
}

\author{
IMPLICATIONS OF THE HEALTH INFORMATION SYSTEM IN THE \\ FAMILYHEALTH STRATEGY: AN INTEGRATIVE REVIEW
}

\author{
Pollyanna Jorge Canuto ${ }^{1}$ \\ Kalyne Araújo Bezerra²
}

\begin{abstract}
RESUMO: Objetivo: Prospectar sobre as implicações dos Sistemas de Informações em Saúde(SI) no âmbito da Atenção Primária em Saúde (APS), avaliar os benefícios e dificuldades encontradas pelos profissionais na utilização do sistema, bem como a inovação trazida por essesserviços e identificar as diferenças entre o SIAB e e-SUS. Método: Reporta-se a uma revisão integrativa através da verificação e análise de artigos oriundos das bases de dados online Biblioteca Virtual em Saúde, pela utilização dos descritores "Sistemas de Informação em Saúde", "Atenção Básica" e "Estratégia de Saúde da Família" combinados pelo uso do operador booleano "and". Foram incluídos nos estudos as publicações com os textos completos, disponíveis em inglês e português, no período de 2015 a 2020. Resultados: Obteve-se 28 documentos científicos e após leitura e exploração do material foram selecionadas 9 publicações que correspondiam ao escopo do estudo, excluindo aqueles que não atendiam aos objetivos e os que se encontravam duplicados. Conclusão: A informação em saúde é considerada um instrumento essencial, que atua na conexão e interoperabilidade das informações relevantes a saúde, além de considerar que o SI traz importantes mudanças para o processo de trabalho da APS, inferindo nas análises em situação de saúde, gerando poder decisório nas ações, atuando como escopo de conhecimentos e impactando diretamente na qualidade da atenção.
\end{abstract}

Palavras chave: Sistemas de Informação em Saúde. Atenção Primária à Saúde. Estratégia Saúde da Família.

ABSTRACT: Objective: To prospect about the implications of Health Information Systems (IS) in the scope of Primary Health Care (PHC), evaluate the benefits and difficulties encountered by professionals in the use of the system, as well as the

\footnotetext{
${ }^{1}$ Graduada em Enfermagem pela UNIFACISA, Mestranda em Saúde Pública pela UEPB.

${ }^{2}$ Graduanda em Enfermagem pela UNIFACISA.
} 
innovation brought by these services and identify the differences between SIAB and e-SUS. Method: Reports an integrative review through the verification and analysis of articles from the online databases Virtual Health Library, using the descriptors "Health Information Systems", "Primary Care" and "Family Health Strategy "Combined using the Boolean operator" and ". Publications with full texts, available in English and Portuguese, between 2015 and 2020 were included in the studies. Results: 28 scientific documents were obtained and after reading and exploring the material, 9 publications were selected that corresponded to the scope of the study, excluding those that did not meet the objectives and those that were duplicated. Conclusion: Health information is considered an essential instrument, which acts in the connection and interoperability of information relevant to health, in addition to considering that the IS brings important changes to the PHC work process, inferring in the analysis of health situations, generating decision-making power in actions, acting as a scope of knowledge and directly impacting the quality of care.

Keywords: Health Information Systems; Primary Health Care; Family Health Strategy. 


\section{INTRODUÇÃO}

A Atenção Primária em Saúde- APS é mencionada como uma mudança no paradigma em saúde, uma vez entendida como um modo de reorganização das práticas, sendo centro de uma rede, e tem como objetivos primordiais: funcionar como porta de entrada preferencial do usuário no sistema, a articulação com outros níveis de atenção e, ao mesmo tempo, regula o fluxo, otimizando os gastos em saúde (BRASIL, 2017). Para Brito e colaboradores (2018), a Estratégia Saúde da Família (ESF) é o modelo preferencial de organização da APS no Brasil, eespera-se que ela seja capaz de abordar o processo de saúde-doença dos indivíduos de modo singular e articulado ao contexto familiar e comunitário.

Em consonância a esta descrição, para que o cuidado seja acompanhado de forma integral e resolutivo, como preconizado pelas diretrizes do Sistema Único de Saúde (SUS), o Departamento de Atenção Básica (DAB) busca o constante aprimoramento dos Sistemas de Informações (SI), instrumentos indispensáveis para averiguar a situação de saúde populacionale a execução dos serviços prestados (RIVEMALES et al., 2017). Desta maneira, com a descentralização de responsabilidades, serviços e recursos, os sistemas de saúde são geridos o mais próximo possível do nível de prestação de serviços (PINHEIRO et al., 2014).

Nesse prospecto, os bancos de dados e Sistemas de Informação em Saúde (SIS) constituem ferramentas importantes para o planejamento e a avaliação das políticas de saúde, assim como dos serviços, redes e sistemas de saúde (FERLA et al., 2012). Dessa maneira, o Sistema de informação usado na atenção básica é uma ferramenta de trabalho essencial na ESF e serve para sistematizar os dados levantados da comunidade da área adscrita, otimizando o trabalho da equipe (LIMA et al., 2012).

Atualmente, o sistema de informação em saúde na APS no Brasil é o SISAB (Sistema de informação em saúde da atenção básica) que é alimentado pelo dispositivo de software chamado e-SUS, onde possui duas formas de provimento 
dos dados: a coleta de dadossimplificada (CDS) intenta informações individualizadas por cidadão, justificados pela quantidade reduzida de fichas e dinamismo nos relatórios de saúde; e o prontuário eletrônico do cidadão (PEC) que evidencia tecnologia avançada, pois permite a interoperabilidade com outros sistemas, ofertando suporte clínico e melhor integração dos serviços (BRASIL, 2014).

Assim, considerando a deliberação ocorrida na Comissão Intergestores Tripartite (CIT), em 25 de abril de 2013, resolve: Art. $1^{\circ}$ Fica instituído o Sistema de Informação em Saúde paraa Atenção Básica (SISAB). Art. $2^{\circ} \mathrm{A}$ operacionalização do SISAB será feita por meio da estratégia do Departamento de Atenção Básica (DAB/SAS/MS) denominada e-SUS na APS. (BRASIL, 2013).

Todavia, a utilização das informações torna-se essencial na área de saúde, contudo sua eficácia está diretamente ligada à sua usabilidade sendo necessário que todos os profissionais envolvidos na implantação e utilização de um sistema se apropriem dos mecanismos de uso e conscientizem de sua importância para a rede de serviços. Carreno e colaboradores (2015)endossa que a educação permanente, promoveria efetividade do SI na ESF, bem como a avaliação constante das dificuldades de sua utilização como um instrumento para a programação do trabalho da equipe de saúde.

Nesse sentido, a pertinência de investigar benefícios e dificuldades diante de sua implantação, aspectos inerentes ao processo de inovação, além das diferenças quanto ao SIAB, reportam uniformidade e confiabilidade, arraigando o instrumento de registro, processamento e coleta de dados e a sua dimensão dentro do processo de trabalho. Sendo assim: Quais as implicações dos SI no âmbito da ESF? Desse modo, busca-se prospectar as implicações dos Sistemas de Informação em Saúde no âmbito da APS de forma holística e pertinente ao escopodo estudo. 


\section{MÉTODO}

Para a obtenção dos resultados, optou-se pelo método de revisão integrativa da literatura, de abordagem qualitativa e de caráter exploratório, com o objetivo de compreender melhor as implicações dos Sistemas de Informação em Saúde no âmbito da APS. Para a construção da revisão integrativa o estudo foi realizado seguindo a sequência de três etapas distintas, as quais serão relacionadas a seguir:

A primeira etapa consiste na pesquisa inicial. A consulta foi realizada na BibliotecaVirtual da Saúde - BVS. Para a pesquisa foram determinados e utilizados para cruzamento os seguintes descritores disponíveis no DeCS - Descritores em Ciências da Saúde: 1) "Sistemas de informação em Saúde" AND 2) "Atenção Básica" AND 3) "Estratégia de Saúde da Família".

A segunda etapa consiste na aplicação dos critérios de inclusão e exclusão. Além dos descritores, foram estabelecidos limites para a pesquisa, adotando-se como critérios de inclusão: disponibilidade de texto completo, artigos publicados nos últimos cinco anos e disponíveis nos idiomas português e inglês. Foram excluídos os artigos repetidos entre as bases de dados e os que não se enquadram nos objetivos da pesquisa.

Inicialmente, a busca expôs 143 artigos na BVS. Após aplicação dos filtros, restaram 28 artigos e após foi realizada leitura de seus respectivos títulos e resumos. Foram aceitos os artigos que respondiam à pergunta norteadora: Quais as implicações dos SI no âmbito da ESF? Em seguida, procedeu-se a leitura dos artigos completos, dentre aqueles que se enquadraram nos critérios. Desta forma, ao final da seleção, foram selecionados 9 artigos na BVS.

A terceira etapa refere-se a revisão dos artigos. Para a análise do material coletado, dois revisores leram criticamente de maneira independente os artigos selecionados, extraindo as unidades de interesse para o estudo. As discordâncias foram resolvidas por consenso entre os autores. 


\section{RESULTADOS}

Obteve-se como resultado 28 publicações e após a leitura e exploração do material foram selecionadas 9 publicações, sendo composta por oito artigos e uma dissertação.

A base de dados em que mais houve publicações sobre o tema foi a LILACS com oito publicações, e o ano com menos publicação foi 2020 (ano em andamento) com apenas uma publicação.

Tabela 1 - Descrição dos artigos selecionados para compor o estudo.

\begin{tabular}{|c|c|c|c|c|c|c|c|}
\hline $\mathbf{N}^{\circ}$ & Ano & Título & Autore(s) & Objetivo & $\begin{array}{c}\text { Base de } \\
\text { dados }\end{array}$ & Tipo & Revista \\
\hline 1 & 2020 & $\begin{array}{l}\text { Blogs e redes } \\
\text { sociais na } \\
\text { atenção à } \\
\text { saúde da } \\
\text { família: o que a } \\
\text { comunicação } \\
\text { online traz de } \\
\text { novo? }\end{array}$ & $\begin{array}{l}\text { Barcelos, } \\
\text { P.E.L.; Lima, } \\
\text { T.V.; Aguiar, } \\
\text { A.C. de }\end{array}$ & \begin{tabular}{lr} 
Investigar & a \\
criação & de \\
ambientes & de \\
comunicação & \\
online r nos \\
serviços r de \\
atenção primária \\
no município do \\
\multicolumn{2}{l}{ Rio de Janeiro }
\end{tabular} & LILACS & Artigo & $\begin{array}{l}\text { RECIIS } \\
\text { (online) }\end{array}$ \\
\hline 2 & 2018 & $\begin{array}{lr}\text { Difusão } & \text { da } \\
\text { inovação } & \text { e- } \\
\text { SUS Atenção } \\
\text { Básica } & \text { em } \\
\text { Equipes } & \text { de } \\
\text { Saúde } & \text { da } \\
\text { Família } & \end{array}$ & $\begin{array}{l}\text { Silva, T. I. } \\
\text { M.; } \\
\text { Cavalcante, } \\
\text { R.B.; Santos, } \\
\text { R.C. dos; } \\
\text { Gontijo, T. L.; } \\
\text { Guimarães, } \\
\text { E.A. de A.; } \\
\text { Oliveira, V. } \\
\text { C. de. }\end{array}$ & $\begin{array}{l}\text { Analisar a difusão } \\
\text { da inovação e- } \\
\text { SUS Atenção } \\
\text { Básica em } \\
\text { Equipes de Saúde } \\
\text { daFamília }\end{array}$ & LILACS & Artigo & $\begin{array}{l}\text { Revista } \\
\text { brasileira } \\
\text { de } \\
\text { enfermag } \\
\text { em }\end{array}$ \\
\hline 3 & 2018 & $\begin{array}{l}\text { Processo de } \\
\text { implantação } \\
\text { do e-SUS } \\
\text { Atenção } \\
\text { Básica em } \\
\text { Sobral-CE }\end{array}$ & $\begin{array}{l}\text { Ribeiro,M.A.; } \\
\text { Muniz, T. B. } \\
\text { F; } \\
\text { Albuquerque, } \\
\text { I. M. N.; } \\
\text { Vasconcelos, } \\
\text { A. Á.; Costa, } \\
\text { M. M. da; } \\
\text { Vasconcelos, } \\
\text { A.M. B. }\end{array}$ & $\begin{array}{lr}\text { Conhecer } & \text { os } \\
\text { desafios } & \text { da } \\
\text { implantação do e- } \\
\text { SUS Atenção } \\
\text { Básica no } \\
\text { programa } \\
\text { Estratégia de } \\
\text { Saúde da Família } \\
\text { do município } \\
\text { de Sobral-CE }\end{array}$ & LILACS & Artigo & $\begin{array}{l}\text { RECIIS } \\
\text { (Online) }\end{array}$ \\
\hline 4 & 2018 & $\begin{array}{ll}\text { Difusão } & \text { da } \\
\text { inovação } & \\
\text { tecnológica } & \text { e- } \\
\text { SUS } & A B \text { : } \\
\text { aceitação ou }\end{array}$ & $\begin{array}{l}\text { Silva, T. I. } \\
\text { M.; } \\
\text { Cavalcante, } \\
\text { R. B.; Silva, } \\
\text { H. R. M. da; }\end{array}$ & $\begin{array}{l}\text { Analisar a difusão } \\
\text { da inovação } \\
\text { tecnológica } \\
\text { "estratégia e-SUS } \\
\text { AB" em uma }\end{array}$ & $\begin{array}{l}\text { BDENF } \\
- \\
\text { Enferma } \\
g \text { em e } \\
\text { LILACS }\end{array}$ & Artigo & $\begin{array}{l}\text { Cogitare } \\
\text { enfermag } \\
\text { em }\end{array}$ \\
\hline
\end{tabular}




\begin{tabular}{|c|c|c|c|c|c|c|c|}
\hline & & rejeição? & $\begin{array}{l}\text { Santos, R. } \\
\text { C.; } \\
\text { Guimarães, } \\
\text { E. A. de A.; } \\
\text { Pinheiro, M. } \\
\text { M. K. }\end{array}$ & $\begin{array}{l}\text { equipe de saúde } \\
\text { dafamília do oeste } \\
\text { deMinas Gerais }\end{array}$ & & & \\
\hline 5 & 2017 & $\begin{array}{l}\text { O E-SUS } \\
\text { atenção } \\
\text { básica e a } \\
\text { coleta de } \\
\text { dados } \\
\text { simplificada: } \\
\text { relatos da } \\
\text { implementaçã } \\
\text { O em uma } \\
\text { estratégia } \\
\text { saúde da } \\
\text { família }\end{array}$ & $\begin{array}{l}\text { Medeiros, J. } \\
\text { B.; Holmes, } \\
\text { E. S.; } \\
\text { Albuquerque, } \\
\text { S. G. E. de; } \\
\text { Santos, S. R. } \\
\text { dos. }\end{array}$ & $\begin{array}{l}\text { Relatar os } \\
\text { problemas e as } \\
\text { facilidades } \\
\text { vivenciadas pelos } \\
\text { profissionais da } \\
\text { estratégia saúde } \\
\text { da família na } \\
\text { implementação } \\
\text { doE-SUS Atenção } \\
\text { Básica }\end{array}$ & LILACS & Artigo & $\begin{array}{l}\text { Revista } \\
\text { deAPS }\end{array}$ \\
\hline 6 & 2017 & $\begin{array}{l}\text { Difusão da } \\
\text { inovação e- } \\
\text { sus atenção } \\
\text { básica (e-sus } \\
\text { ab) emequipes } \\
\text { de saúde da } \\
\text { família }\end{array}$ & Silva, T.I.M. & $\begin{array}{l}\text { Analisar a difusão } \\
\text { da inovação e- } \\
\text { SUS AB em } \\
\text { equipes de saúde } \\
\text { da família da } \\
\text { região Oeste de } \\
\text { Minas Gerais. }\end{array}$ & $\begin{array}{l}\text { BDENF } \\
- \\
\text { Enferma } \\
\text { gem }\end{array}$ & $\begin{array}{l}\text { Disserta } \\
\text { ção }\end{array}$ & \\
\hline 7 & 2015 & $\begin{array}{l}\text { Indicadores e } \\
\text { informações } \\
\text { no } \\
\text { planejamento } \\
\text { local em } \\
\text { saúde: visão } \\
\text { dos } \\
\text { enfermeiros } \\
\text { da estratégia } \\
\text { saúde da } \\
\text { família }\end{array}$ & $\begin{array}{l}\text { Senna,M.H.; } \\
\text { Andrade,S.R. } \\
\text { de. }\end{array}$ & $\begin{array}{lr}\text { Analisar } & \text { a } \\
\text { utilização dos } & \text { indicadores de } \\
\text { saúde do Sistema } \\
\text { de Informação da } \\
\text { Atenção Básica } \\
\text { pelos enfermeiros } \\
\text { da Estratégia } \\
\text { Saúde da Família } \\
\text { no planejamento } \\
\text { das ações locais } \\
\text { em saúde. }\end{array}$ & $\begin{array}{l}\text { BDENF } \\
- \\
\text { Enferma } \\
\text { gem e } \\
\text { LILACS }\end{array}$ & Artigo & $\begin{array}{l}\text { Texto \& } \\
\text { contexto } \\
\text { enferm }\end{array}$ \\
\hline 8 & 2015 & $\begin{array}{l}\text { Percepções } \\
\text { sobre } \\
\text { instrumentos } \\
\text { de coleta de } \\
\text { um Sistema } \\
\text { delnformação }\end{array}$ & 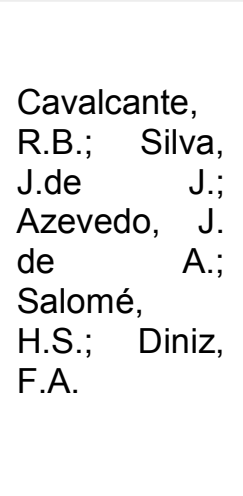 & $\begin{array}{ll}\text { Analisar como os } \\
\text { profissionais da } \\
\text { Estratégia de } \\
\text { Saúde da Família } \\
\text { (ESF) percebem } \\
\text { os instrumentos } \\
\text { de coleta do } \\
\text { Sistema d de } \\
\text { Informação da } \\
\text { Atenção } \\
\text { Básica (SIAB) e } \\
\text { as utilizam }\end{array}$ & LILACS & Artigo & $\begin{array}{l}\text { J. health } \\
\text { inform }\end{array}$ \\
\hline
\end{tabular}




\begin{tabular}{|c|c|c|c|c|c|c|c|}
\hline 9 & 2015 & $\begin{array}{l}\text { Percepção e } \\
\text { avaliação dos } \\
\text { enfermeiros } \\
\text { das equipes } \\
\text { do programa } \\
\text { saúde } \\
\text { relacionado ao } \\
\text { sistema de } \\
\text { informação da } \\
\text { atenção } \\
\text { básica (SIAB) }\end{array}$ & $\begin{array}{l}\text { Ferreira, H.; } \\
\text { Lala, E. R. } \\
\text { P.; Cabral, P. } \\
\text { P.; Sobrinho, } \\
\text { R. A.da S. }\end{array}$ & $\begin{array}{l}\text { Detectar as } \\
\text { dificuldades } \\
\text { encontradas } \\
\text { pelos Enfermeiros } \\
\text { do PSF, no } \\
\text { município de Foz } \\
\text { do Iguaçu-PR, ao } \\
\text { manusear oSIAB }\end{array}$ & LILACS & Artigo & $\begin{array}{l}\text { Revista } \\
\text { deAPS }\end{array}$ \\
\hline
\end{tabular}

Fonte: Dados da Pesquisa, 2020.

\section{DISCUSSÃO}

Diante do processo de informatização é possível identificar a facilidades/dificuldades no acesso e na forma de implantação, além disso, a importância para a assistência prestada ao usuário do serviço, como também os dois sistemas ofertados, o SIAB e o e-SUS, possuem pontos a serem aprimorados de acordo com os profissionais e com a realidade abordada.

Utilizando os meios de informática como aliados, no Rio de Janeiro, a secretaria municipal de saúde criou blogs com a intenção de aproximar a população aos serviços de saúde, bem como registrar atividades da APS. Essa rede de blogs possui como valores o profissionalismo, a velocidade de informação, a interatividade entre os usuários e profissionais, focando nos resultados obtidos na assistência, bem como, vigilância e promoção da saúde, e a troca de experiências entre os profissionais (BARCELOS et al., 2020).

As contribuições da implantação dos SIS na rede da APS, principalmente o eSUS, interferem diretamente na assistência à comunidade por meio da individualização do usuário, sendo possível o profissional no momento do atendimento ter acesso ao histórico do paciente, sua condição social e econômica, entre outros (MEDEIROS et al., 2017; CAVALCANTE et al., 2018), informações estas que, podem ser acessadas por diferentes equipes de saúde (SILVA,2017).

Sendo assim, é possível um melhor acompanhamento de grupos prioritários como diabéticos, hipertensos, gestantes, etc. (SILVA et al., 2018), permitindo o 
planejamento da equipe de saúde para uma melhor organização do trabalho (CAVALCANTE et al., 2018), gerando um atendimento direcionado ao paciente e focando nas suas necessidades(MEDEIROS et al., 2017).

Ao processo de implantação do $\mathrm{Sl}$, houveram diversos anseios e questionamentos por parte dos profissionais (MEDEIROS et al., 2017; CAVALCANTE et al., 2015) que alegaram não terem sido treinados para a utilização das fichas e manuseio do programa (FERREIRA et al., 2015). De acordo com Medeiros e colaboradores (2017), a dificuldade encontrada de início consistiu no acesso ao registro dos usuários, pois é necessário o cartão do SUS que, muitas vezes era esquecido pelos pacientes sendo necessário a conscientização da população e a realização de novos cadastros.

A falta de internet e de computadores para a utilização do software também é relatada pela equipe de saúde, em decorrência disso há a sobrecarga de trabalho, pois muitas vezes o profissional médico não realiza a digitalização de suas produções, sendo direcionadas a outros profissionais, o que gera uma assistência prejudicada e aumenta o tempo de espera dos pacientes (SILVA, 2017; RIBEIRO et al., 2018). Diante do explanado, o sigilo, a privacidade e a confidencialidade das informações dos usuários ficam comprometidas, mediante o profissional da consulta não ser o mesmo que realiza a alimentação do sistema, podendo interferir no processo. Todavia, esses dados precisam ser postos no sistema, pois são importantes para a solicitação de recursos do município (CAVALCANTE et al., 2018; SENNA, ANDRADE, 2015).

Com relação ao SIAB, foi visto como um sistema utilizado para o planejamento de ações da equipe de saúde, porém não é possível realizar uma intervenção direta com base nesses dados, pois os dados mostrados são de nível nacional. Há uma limitação do registro de algumas informações como o uso de drogas e exames preventivos de câncer uterino, por exemplo. O cadastramento dos usuários é realizado pelos Agentes Comunitários de Saúde (ACS), que na maioria das vezes não é alimentado pela falta destes profissionais (FERREIRA et al., 2015; SENNA, ANDRADE, 2015).

As sugestões de melhoria indicadas pelos profissionais são descritas em: inclusão de dados e informações sobre a população da área da ESF, o relatório 
específico de cada região e a ampliação de alimentação dos dados por todos os profissionais da APS, como os odontólogos e psicólogos, por exemplo (SENNA; ANDRADE, 2015).

O e-SUS é o sistema que veio como substituição para o SIAB, tido como mais completo em comparação aos anteriores com o objetivo de conhecer o perfil epidemiológico e traçar metas a partir deles (RIBEIRO et al., 2018). Porém, ainda assim há o preenchimento de fichas de outros sistemas o que gera um acúmulo destas para a digitalização (CAVALCANTE et al., 2015). Com isso, a principal sugestão para melhoria da utilização é dada pela ocorrência de oficinas para treinamento dos profissionais para adaptá-los ao novo sistema (RIBEIRO et al., 2018).

Destarte, os avanços tecnológicos são vistos como imprescindíveis na área da saúde, pois além dos benefícios, e excelente resposta na luta contra as doenças, traz consigo uma gama de conhecimentos novos a cada instante para os profissionais de saúde. Por este contexto e relevância apresentados, os profissionais de saúde devem ter acesso à essas informações, aos dados extraídos nos sistemas, mostrando uma realidade sanitária e, portanto, podem e devem ser usados para o planejamento de intervenções (SILVA et al., 2018).

Nessa perspectiva, considera-se importante que os gestores possam escutar os profissionais das equipes de saúde com a finalidade de identificar as melhorias a serem realizadas para garantir a fidedignidade dos dados coletados e informações produzidas, tendo em vista que a qualidade das informações interfere diretamente nas ações em saúde desenvolvidas.

\section{CONCLUSÕES}

A informação em saúde é tida como um instrumento essencial para o conhecimento da realidade socioeconômica e epidemiológica local, servindo de apoio ao processo decisório e planejamento da gestão em saúde no SUS. Indubitavelmente, o sistema de informações em saúde é uma estratégia do 
Ministério da Saúde através do Departamento de Atenção Básica (DAB) para reestruturar as informações da APS, modernizando sua plataforma tecnológica como objetivo de informatizar as unidades básicas de saúde, oferecendo ferramentas para ampliar o cuidado e melhorar o acompanhamento da gestão.

Diante disso, ficou abordado neste estudo algumas facilidades, diferentes dificuldades, variados pontos de vista sobre a implantação, e sobretudo diversas implicações no processo detrabalho dos profissionais da ESF.

No entanto, ainda existem muitas lacunas para um bom funcionamento do sistema para que o mesmo consiga funcionar com preparo, como descritas nesta pesquisa. Entretanto, a estratégia e-SUS é uma ferramenta inovadora que preconiza a integração e interoperabilidade, com vistas a eliminação de retrabalhos e produção de dados confiáveis. Além destes pontos, foi demonstrado que a estratégia de implantação deste sistema busca melhorar toda da estrutura detrabalho das equipes da ESF e como consequência melhorar o serviço prestado à população.

Por conseguinte, é de extrema importância a promoção de ações para o aprimoramento dos sistemas, além de promover capacitação de profissionais de saúde para uma melhor consciência da importância dos SIS como ferramenta de vigilância epidemiológica, processo decisório, análise e planejamento situacional e escopo da saúde.

\section{REFERÊNCIAS BIBLIOGRÁFICAS}

BARCELOS P.E.L., LIMA T.V., AGUIAR A.C. Blogs e redes sociais na atenção à saúde da família: o que a comunicação online traz de novo?. Revista Eletrônica de Comunicação, Informação e Inovação em Saúde, [s.I.], v. 14, n. 1, p. 126-149, 31 mar. 2020. Instituto de Comunicacao e Informacao Cientifica e Tecnologica em Saude. http://dx.doi.org/10.29397/reciis.v14i1.1747.

BRASIL. DATASUS [Internet]. Legislação de saúde, 2013. Disponível em: http://bvsms.saude.gov.br/bvs/saudelegis/gm/2013/prt1412_10_07_2013.html. Acesso em:08 de dezembro de 2019.

BRASIL. MINISTÉRIO DA SAÚDE. Manual do Sistema com Coleta de Dados Simplificada - CDS, versão preliminar. Brasília - DF. 2014.

BRASIL. Portaria $n^{\circ} 2.436$, de 21 de setembro de 2017. Aprova a Política Nacional de Atenção Básica, estabelecendo a revisão de diretrizes para a organização da Atenção Básica, no âmbito do Sistema Único de Saúde (SUS). Brasília, Disponível em: 
https://bvsms.saude.gov.br/bvs/saudelegis/gm/2017/prt2436_22_09_2017.html. Acesso em:21 abr. 2020.

BRITO G.E.G., MENDES A.C.G., NETO P.M.S. O objeto de trabalho na Estratégia Saúde da Família. Interface Comunic Saúde Educ. 2017; 22(64):77-86.

CARRENO I., MORESCHI C., MARINA B., HENDGES D.J.B., REMPEL C., OLIVEIRA M.M.C. Análise do uso de dados do Sistema de Informação de Atenção Primária à Saúde(SIAB): revisão integrativa da literatura. Ciênc Saúde Coletiva [Internet]. 2015; [citado em 13/11/2017]; 20 (3): 947-56. Disponível em: https: //dx.doi/ 10.1590/141381232015203.17002013.

CAVALCANTE R.B., SILVA H.R.M., SILVA T.I.M., SANTOS R.C., GUIMARÃES E.A.A., PINHEIRO M.M.K. DIFUSÃO DA INOVAÇÃO TECNOLÓGICA e-SUS AB: aceitação ou rejeição?.Cogitare Enfermagem, [s.I.], v. 23, n. 3, p. 1-11, 16 out. 2018. Universidade Federaldo Parana. http://dx.doi.org/10.5380/ce.v23i3.55911.

CAVALCANTE R.B., SILVA J.J., AZEVEDO J.A., SALOMÉ H.S., DINIZ F.A. Percepções sobre instrumentos de coleta de um Sistema de Informação. J. Health Inform. Minas Gerais, p. 1622. jan. 2015.

FERLA A.A., CECIM R.B., ALBA R.D. Information, education and health care work: Beyond evidence, collective intelligence. RECIIS - Rev Eletr Com Inf Inov Saude [Internet]. 2012Ago. Disponível em:

https://www.researchgate.net/publication/314814792_Information_education_and_health_car e_work_Beyond_evidence_collective_intelligence_-_DOI_103395reciisv6i2Sup1620en>.

Acesso em: 02 mai 2020.

FERREIRA, Helder; LALA, Eliane Raquel Peres; CABRAL, Priscila Paiva; SILVA SOBRINHO, Reinaldo Antônio da. PERCEPÇÃO E AVALIAÇÃO DOS ENFERMEIROS DAS EQUIPES DO PROGRAMA SAÚDE RELACIONADO AO SISTEMA DE INFORMAÇÃO DA ATENÇÃO BÁSICA (SIAB). Revista Atenção Primária A Saúde, Paraná, v. 18, n. 1, p. 70-77, mar. 2015.

LIMA A. P., CORREAA A. C. P., OLIVEIRA Q. C. Conhecimento de Agentes Comunitários de Saúde sobre os instrumentos de coleta de dados do SIAB. Ver. Bras. Enferm. 2012; 65(1):121127.

MEDEIROS J.B., HOLMES E.S., ALBUQUERQUE S.G.E., SANTOS S.R., CANDEIA R.M.S., COSTA T. O E-SUS ATENÇÃO BÁSICA E A COLETA DE DADOS SIMPLIFICADA: relatos da implementação em uma estratégia saúde da família. Revista de Aps, [s.I.], v. 20, n. 1, p. 145149, 31 jul. 2017. Universidade Federal de Juiz de Fora. http://dx.doi.org/10.34019/18098363.2017.v20.15784.

PINHEIRO, Alba Lúcia Santos; MARTINS, Aline Freitas Paraíso; PINTO, lone Carvalho; SILVA, Dejeane de Oliveira; ZACHARIAS, Fabiana Costa Machado; GOMIDE, Mariana Figueiredo Souza. Utilização dos Sistemas de Informação: Desafios para a Gestão da Saúde. Ciência, Cuidado e Saúde, Paraná, v. 14, n. 3, p. 1307-1314, jul./set. 2015. Disponível em: http://periodicos.uem.br/ojs/index.php/CiencCuidSaude/article/view/24356. Acesso em: $13 \mathrm{dez}$. 2019.

RIBEIRO M.A., MUNIZ T.B.F., ALBUQUERQUE I.M.N., VASCONCELOS A.A., COSTA M.M., VASCONCELOS A.M.B. Processo de implantação do e-SUS Atenção Básica em Sobral - CE. Revista Eletrônica de Comunicação, Informação e Inovação em Saúde, [s.l.], v. 12, n. 3, p. 258-267, 25 set. 2018. Instituto de Comunicacao e Informacao Cientifica e Tecnologica em Saude. http://dx.doi.org/10.29397/reciis.v12i3.1364.

RIVEMALES M.C.C., SANTOS D.G.R., SANTOS R.N.S. Impressões de estagiárias de enfermagem quanto a implantação de um sistema de informações na atenção básica. Revista Baiana de Saúde Pública, [S.I.], v. 41, n. 1, dez. 2017. ISSN 2318-2660. Disponível em: 
<http://rbsp.sesab.ba.gov.br/index.php/rbsp/article/view/2377>. Acesso em: 10 dez. 2019.

SENNA M.H., ANDRADE S.R. Indicators and information in local health planing: theperspective of the family health strategy nurses. Texto \& Contexto - Enfermagem, [s.I.], v. 24, n. 4, p. 950-958, dez. 2015. FapUNIFESP (SciELO). http://dx.doi.org/10.1590/01040707201500004340014.

SILVA T.I.M. DIFUSÃO DA INOVAÇÃOe-SUS ATENÇÃO BÁSICA (e-SUS AB) EM EQUIPES DE SAÚDE DA FAMÍLIA. 2017. 142 f. Dissertação (Mestrado) - Curso de Enfermagem, Universidade Federal de SÃo JoÃo del Rei, Divinópolis, 2017.

SILVA T.I.M., CAVALCANTE R.B., SANTOS R.C., GONTIJO T.L., GUIMARÃES E.A.A., OLIVEIRA V.C. Diffusion of the e-SUS Primary Care innovation in Family Health Teams. Revista Brasileira de Enfermagem, [s.I.], v. 71, n. 6, p. 2945-2952, dez. 2018. FapUNIFESP (SciELO). http://dx.doi.org/10.1590/0034-7167-2018-0053. 